\title{
Economical Justification of Flexible Microassembly Cells
}

\author{
S. Koelemeijer Chollet, F. Bourgeois, J. Jacot \\ sandra.koelemeijer@epfl.ch \\ Laboratoire de Production Microtechnique, Institut de Production et Robotique \\ Ecole Polytechnique Fédérale de Lausanne, CH-1015 Lausanne, Switzerland
}

\begin{abstract}
Cost is a most important factor to take into account when choosing an assembly system. Traditional choice between manual and automatic assembly with several degrees of partial automation is no longer possible in microassembly. Automation is crucial for precision and quality in handling and attachment of microcomponents. We show the economical interest of a very flexible semiautomatic assembly cell, and when it is most cost-effective. We propose a realization based on a high precision robot and an Intuitive Programming Interface.
\end{abstract}

\section{Introduction}

Assembly cost is often prohibitive for microsystem products unless they are mass produced. It represents on average $50 \%$ of total product costs, and significantly more for small batches [1] [2]. Only few microsystem products are mass produced, in that case, production tools including the assembly line, are product dedicated and highly automated. However most microsystems are application dedicated and therefore produced in small quantities. They are not economically viable and do not succeed on the market. Automation is necessary in microsystem assembly to achieve the required precision. The cost of manual assembly rapidly becomes excessive as the size of components reduces, not only due to longer cycle times, but also to human errors and low component yield. The major problem is to write off expensive and dedicated high precision equipment on small production quantities.

On one hand, industry proposes traditional assembly cells, with big robots and very rigid frames. Assembly installations became bigger, heavier, and more complex for the production of smaller and smaller products. On the other hand, research institutes try to find solutions to this anomaly by developing tiny robots with high precision movements. These robots will be integrated in small desktop nanofactories [3] [4], like the one proposed by Gaugel [5] (Fraunhofer IPA). Research in this field is just start- ing. We propose a step by step progress, starting with the flexibility aspect and the modularity of the assembly equipment. We think these two points are crucial and very promising. In this paper, we show that flexible and partial automation is particularly interesting for small and medium sized batches and production quantities. We analyze the variation of production cost versus automation degree and batch size. We show for which product size this kind of assembly cells are the most appropriated, and pin point the transition from micro to macro in the respect of optimal equipment cost effectiveness. Finally we propose a semi-automatic flexible and modular microassembly cell, based on a high precision robot and a simple programming interface developed for non qualified operators, called IPI ('Intuitive Programming Interface").

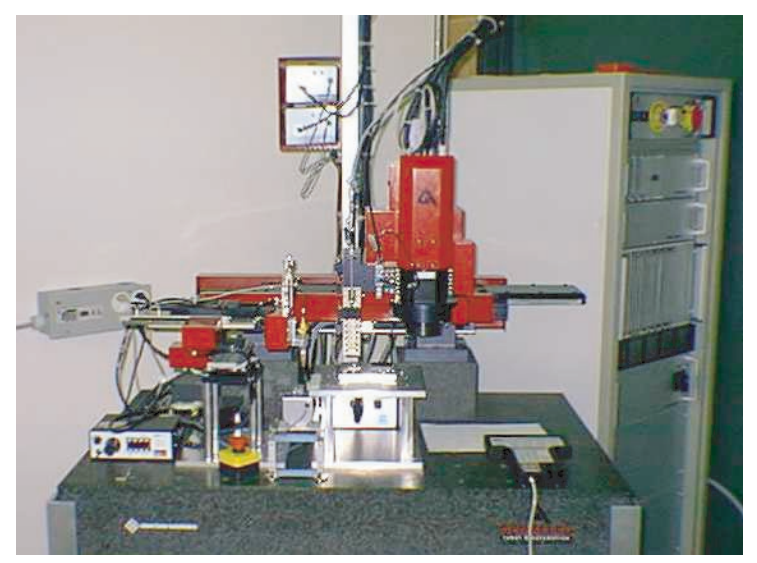

Figure 1: Microassembly station of the LPM-EPFL

\section{Assembly cost models}

\subsection{Interest of cost models}

A condition of product success is a proper cost value balance. Therefore cost models are so important. They 
allow the correct choice of shop floor configuration for a specific product, based on quantitative criteria. In the end, product cost is the only objective criteria [6] [7]. We first propose an assembly cost model, and then compare the costs of semi automatic and manual assembly for two different product categories. Watch industry type products (i.e. products where the component size is at least $2 \mathrm{~mm}$, with an assembly precision of $10 \mu \mathrm{m}$ ), and microsystem products (with components of less than 2 to $6 \mathrm{~mm}$ and an assembly precision of $1 \mu \mathrm{m}$ ). This demonstrates the interest of a semi-automatic assembly cell for microassembly. We also compare the assembly costs of a microsystem product in three different assembly systems. First we discuss the cost of each assembly operation, and then the total assembly cost, taking into account the batch size and component yield. We make the assumption that the equipment is used to its full capacity, which is, of course, too optimistic. The three different cases we consider are:

- Manual assembly by an operator equipped with binoculars and tweezers. The operator effectuates the assembly of all components.

- Assembly on a flexible semi-automatic microassembly cell. The operator places the components and prepares the assembly sequence with the help of an Intuitive Programming Interface. After that, the cell operates independently and fully automatically, and assembles all components. Set-ups are very short, less than 1 minute for products the system is familiar with, 5 minutes for a new product [8].

- Automatic assembly on a dedicated assembly line, with one component per assembly station. The operator feeds the components, the assembly line operates fully autonomously. Cycle times are very short, each of the stations being specialized. Batch and product changes are relatively long on this type of installation, because more or less important adjustments are necessary and new products require new adjustments and programming by engineers or technicians.

\subsection{Analysis of the assembly operation costs}

The assembly of a component consists of two steps: component supply, that consists of feeding, separation and orientation, and the proper assembly operation, that is placement and attachment. We will here consider placement and attachment as one operation; it is not possible to dissociate these two steps in microassembly as the component is not stable prior to the attaching procedure [9].

Feeding An operator feeds the components onto the assembly cell at the determined feeding spot: pouring the components into a vibrating bowl or funnel, placing of pallets, strips, or wafers. The cost of this operation depends on the intervention time $T_{\text {feed }}$ of the operator, the hourly labour rate of the operator $c_{h}$, and the number of components fed in at one time $S_{\text {feed }}$. The latter depends either on the space available on the assembly cell or on the batch size $S$.

$$
c_{\text {op feed }}=\frac{T_{\text {feed }} \cdot c_{h}}{\min \left(S, S_{\text {feed }}\right)}
$$

Separation and orientation of the component The component must be separated from the bulk of components, and oriented for assembly, that is, for the picking by the gripper. A vibrating bowl is mostly used for separation and orientation in the case of automatic assembly of relatively big sized components. Smaller components are fed in on strips, pallets or directly on wafers in the case of microsystems. In manual assembly, the operator carries out these operations while handling the component for placing [10]. The cost $c_{\text {op auto orientation }}$ of the orientation operation in automatic assembly is the equipment write off cost $C_{\text {equip orient }}$ divided by the number of cycles $Q_{\text {cycles }}$ :

$$
c_{\text {op auto orientation }}=\frac{C_{\text {equip orient }}}{Q_{\text {cycles }}}
$$

The manual orientation time $T_{\text {or }}$ is given by Boothroyd: it depends on the form of the component, on the manipulation difficulty factor, and on its size. Manipulation times given are for components up to the size of watch industry components; for smaller components and a higher precision assembly, no systematic research has been done. The cost of the manual orientation operation is:

$$
c_{\text {op } \text { man orientation }}=T_{\text {or }} \cdot c_{h}
$$

Placement and attachment of the component The automatic assembly operation is a typical pick and place operation, its $\cos t c_{O p}$ auto assembly depends on the equipment cost and the number of cycles on which the equipment is written off:

$$
c_{\text {op aut assembly }}=\frac{C_{\text {equip }}}{Q_{\text {cycles }}}
$$

In manual assembly, the cost essentially depends on the operation cycle time $T_{c}$ and the hourly labour rate. The manual cycle time is given by Boothroyd. It depends on several parameters, those in particular relative to microassembly are: the manipulation difficulty factor, the fragility of the component, and the fact that tweezers have to be used. In addition, the work station and tool cost, including a microscope for the very small components, must be taken into 
account, which gives an operation cost for manual assembly of:

$$
c_{\text {op man assembly }}=T_{c} \cdot c_{h}+\frac{C_{\text {equip }}}{Q_{\text {cycles }}}
$$

In microassembly, attachment takes on more importance as it becomes more difficult to effectuate [11]: less clipping, more gluing which needs curing time (components must be held down until the position is securely fixed, the risk of movement being too important). For the rest of our analysis we will combine the two assembly operations by adding their cycle times. In a big sized assembly process, opportunities for parallel operations result in shorter cycle times.

\subsection{The influence of component size}

Yield is the rate of conformity of produced parts. Yielded costs have been extensively developed for the microelectronic industry. A good review was performed by Becker [12]. We will here consider the yield of the assembly operation and that of the components.

Assembly yield Defects must be taken into account. If the typical operator performance is $2 \%$ defects for normal sized assembly, it is dramatically lower for operations under a microscope, where the success rates or yields $Y_{\text {ass }}$ are on average around 70\% (manual assembly under microscope of microsystems). The yield is considerably higher with the use of automatic equipment and a well mastered process, and depends less on the component size than for manual assembly.

Component yield The component conformity rate $Y_{\text {comp }}$ obtained by microsystems manufacturing processes is much lower than those of macrocomponents. Yields obtained during traditional manufacturing processes are close to $100 \%$, normally a few tens of ppm.

However, yields are considerably lower for microstructuring processes used for microsystems on wafers. For well known processes as CMOS and high production volumes, conformity rates or yields of $80 \%$ or $90 \%$ are attainable. They mostly depend on the component surface, the integration of sensors and electronics on the same circuit increases its surface, and thus the risk of defects. In microsystems, processes used to produce sensors are often incompatible with those used for microelectronics. Furthermore, batches are small, which again increases the risk of defects. Therefore, yields of $70 \%$ are considered to be very good, and yields of $50 \%$ are common [13].

In general, it is impossible to test the components prior to assembly. They have to be assembled and encapsulated to be testable. The feeding and assembly cost of defective components must be written off on the good components. The manufacturing and assembly cost of a batch of conform microsystem products is thus increased by $25 \%$ to $50 \%$, which does not help the economical success of this type of product. Mastering the manufacturing processes is necessary to guarantee their industrial success.

The quantity of conform products $Q$ is thus inferior to the quantity of effectuated assembly cycles $Q_{\text {cycles }}$ :

$$
Q=\frac{1}{Y_{\text {comp }}} \cdot \frac{1}{Y_{\text {ass }}} \cdot Q_{\text {cycles }}
$$

Thus, to obtain a batch of a given size $S$, a larger batch $S_{\text {cycles }}$ has to be manufactured to take into account the rate of defects.

$$
S=\frac{1}{Y_{\text {comp }}} \cdot \frac{1}{Y_{\text {ass }}} \cdot S_{\text {cycles }}
$$

\subsection{Influence of batch size}

A change of batch will take more or less time according to the type of assembly installation. Two types of changeover time have to be considered, hidden time where the operator can prepare components for the new batch while the operation is in progress, and the set-up time $T_{\text {set-up }}$ requiring complete stoppage of the assembly equipment. The latter results in loss of profit and important costs, because it decreases the number of cycles possible during the total production time $T_{\text {prod }}$. In particular, this is the case when dedicated non flexible equipment is used, or where the stoppage for new product introduction is counted in hours, or even days.

$$
Q_{\text {cycles }}=\frac{T_{\text {prod }}}{T_{c}+\frac{T_{\text {set-up }}}{S_{\text {cycles }}}}
$$

\subsection{Assembly cost}

Thus, the assembly cost for one component is the sum of its feeding, orientation and assembly cost.

$$
c_{\text {op assembly }}=c_{o p \text { feed }}+c_{o p \text { or }}+c_{\text {op ass }}
$$

We either use the cost of manual assembly, or automatic assembly

$$
\begin{gathered}
c_{\text {op man ass }}=\left(\frac{T_{\text {feed }}}{\min \left(S, S_{\text {feed }}\right)}+T_{\text {or }}+T_{c}\right) \cdot c_{h}+\frac{C_{\text {equip }}}{Q_{\text {cycles }}} \\
c_{\text {op auto ass }}=\frac{T_{\text {feed }} \cdot c_{h}}{\min \left(S, S_{\text {feed }}\right)}+\frac{C_{\text {bowl }}+C_{\text {equip }}}{Q_{\text {cycles }}}
\end{gathered}
$$


The cost for the assembly operation for a given product with $N_{\text {comp }}$ components finally is

$$
c_{\text {product assembly }}=\frac{1}{Y_{\text {ass }} \cdot Y_{\text {comp }}} \cdot N_{\text {comp }} \cdot c_{o p \text { assembly }}
$$

\section{Comparison and analysis}

We will explain hereafter the choice of the numerical values in each case.

\subsection{Cycle time}

Cycle time for manual assembly is given by Boothroyd, for products having a size of $6 \mathrm{~mm}$ or slightly less. An orientation and assembly time of $12 \mathrm{~s}$ is thus obtained for the assembly of watch wheels as those assembled on our assembly cell. For a microsystem under a microscope, we will allow here a cycle time of $30 \mathrm{~s}$.

The automatic cycle consists of picking, measuring, transfer, adjusting, placing, and attaching of the component. The flexible microassembly cell handles generic operations, which are not optimised for one particular type of assembly. It is difficult to achieve a precise placing, and the component needs to be maintained during the attachment. This causes very long average cycle times. We obtain a cycle time of $8 \mathrm{~s}$ for the assembly of watch wheels, for microassembly needing an additional attachment, we must allow a cycle time of at least $15 \mathrm{~s}$.

In the case of highly specialized operations, such as flip chip or die-attach or dedicated watch-assembly, a dramatic reduction of cycle time is achieved. This can be done by performing certain tasks in parallel: measurements and adjusting during positioning, attachment on the next station, etc. Parallelization of tasks is not possible on flexible assembly equipment where sequences are not always identical. Here we will assume a cycle time of $3 \mathrm{~s}$.

\subsection{Investment}

The manual assembly cell consists of a work-bank, a microscope and several fixtures and tools. Investment is low, in the range of 5000 Euros. A microassembly cell as developed at LPM-EPFL costs approximately 200'000 Euros. A dedicated assembly line costs about 100'000 Euros per station, with one station per component to be assembled. The write off period is 3 years. We consider one shift.

\subsection{Set-ups}

We must distinguish two different set-ups: batch changes for products "familiar" to the operator or the equipment, and the introduction of new products. A manual assembly cell only needs the supply of new components and, perhaps, the installation of a new fixture. This can be done in $30 \mathrm{~s}$. In the case of a new product, we count a 5 minutes adaptation time for the operator.

The same holds for the flexible assembly cell. In the case of batch changes for familiar products, the operator has to supply the new components on pallets or wafers, change the grippers, and download the corresponding program. Less than one minute will be needed for these operations. In the case of new product introduction, we aim for a total programming time of less than 5 minutes.

The dedicated assembly line requires more time: installation of component feeders, several adjustments, start-up of the line. We count one hour set-up for batch changes of familiar products, but at least 2 hours per station for the introduction of new products.

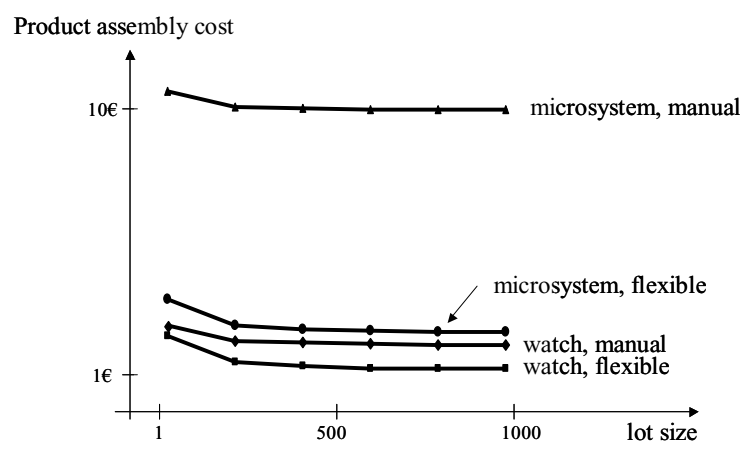

Figure 2: Flexible and manual assembly cost versus lot size for microsystems and watch size components

\subsection{Analysis}

Figures 2 and 3 are obtained by using numerical values above in equation 12. Figure 2 compares the product assembly cost for manual assembly and for assembly on a highly flexible assembly cell, for different batch sizes. The latter is more cost effective in all cases. The difference in total assembly cost is really significant for microsystems, where manual assembly becomes too expensive due to the difficulty for a human operator to perform this type of operations. The system is also advantageous for the assembly of bigger sized products, like watches, but there the advantage is less obvious. Figure 3 compares the cost of the assembly of a microsystem on three different assembly systems: manually, on a highly flexible assembly cell 


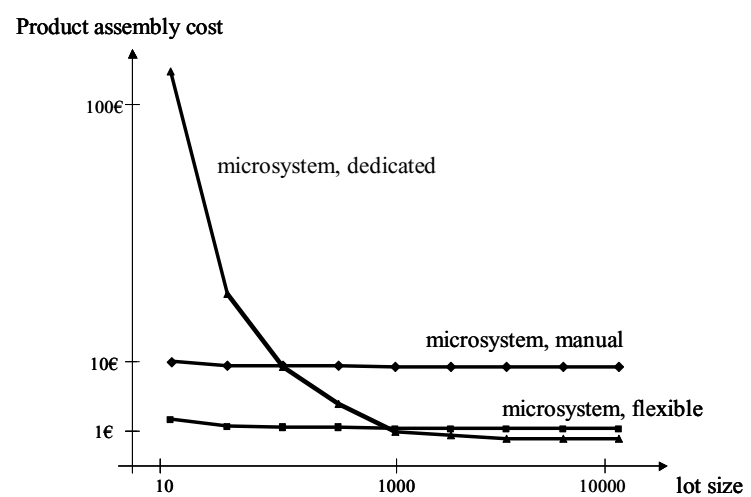

Figure 3: Dedicated, flexible and manual assembly cost versus lot size for microsystems

and on a dedicated assembly line, for batch sizes from 10 to 10 ' 000 parts. It shows that the flexible assembly cell is more cost effective than manual assembly, even for batches as small as 10 parts. Dedicated assembly becomes interesting for batches of about 1000 parts and more, assuming that change-over times remain short.

\section{Proposal for a solution}

At LPM-EPFL, a flexible microassembly cell is developed in accordance with the above characteristics. The cell consists of a high precision robot with a resolution of 0.5 $\mu \mathrm{m}$, and a vision system including zoom and autofocus. The ground surface is approximately $2 \mathrm{~m}^{2}$. The cell can be fitted with a device allowing to pick the components directly on 8" wafer and several grippers. To feed new components, the operator simply needs to install a new wafer or pallet.

Exploitation and programming of the assembly cell are performed by an unqualified operator, and there is no need for either engineers or programmers. An Intuitive Programming Interface allows the operator to view and select the component to assemble and to choose a predefined assembly function such as pick, place, position determination, glue application, etc.

The cell functions in 3 different modes:

- A semi-automatic mode, or teleoperation mode: the operator uses the interface to visualize the parts to be assembled and to indicate the functions to be effectuated, their application points as well as the parameters to be taken into account. This mode is used for very small batches or prototypes.

- A script recording or intuitive programming mode: the operator proceeds as above, but the functions and sequences are registered in a script. This script generates the code for the automatic mode. The final script can be tested step by step, and then safeguarded for immediate or future use.

- Automatic mode: the operator feeds in the working pallets loaded with components to be assembled. The cell functions fully automatically, the script being automatically executed. The operator can follow the operations in real time via the image of camera and a control window. He can carry out other tasks during the automatic assembly time.

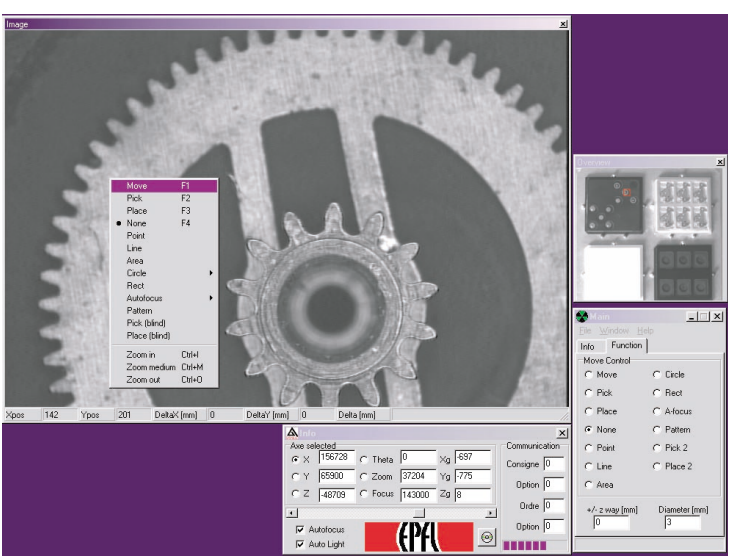

Figure 4: Intuitive Programming Interface

The Intuitive Programming Interface consists of the following elements:

1. A window for live viewing of the process via the camera; this is the main window allowing the operator to visualize the components and to indicate the application points for the selected functions.

2. A window with an image of the working pallet, serving as an active map: this window allows the operator to direct the robot to the components to be handled.

3. A window for the selection of generic functions (move, pick, place).

4. A window for the selection of image processing functions.

5. A window for the control of the robot axes, autofocus and lighting.

6. A database of memorized positions 


\section{Conclusion and future research}

We have demonstrated the interest of the assembly cell for batches of less than 1000 pieces and for production runs with frequent new product introduction. There is an important market for these flexible, modular and compact cells, which can operate at relatively low cost. The customers in this market are mainly microsystem laboratories and SMEs producing small series of high precision products. Another market is that of pre-series: in this case, the machine allows the identification of the main parameters for difficult assemblies prior to heavy investment in assembly equipment for large series. The goal here is to achieve very short "times-to-volume" as product life becomes shorter and less predictable. Our future research on this project will be aimed at the development of a robust, easy to operate, marketable assembly cell.

Further research will also address new attachment techniques in order to reduce cycle times, such a micropressfitting.

\section{References}

[1] G. Beardmore, "Packaging for microengineered devices - lessons from the real world," in Assembly and connections in microsystems, IEE colloquium, 26 February 1997

[2] M. Wilkinson, "The economics of MST." Training in Microsystems, FSRM, Neuchâtel, 2000.

[3] N. Ooyama, "Desktop machining microfactory," in 2nd International Workshop on Microfactories, Fribourg, Switerland, 2000.

[4] J. Breguet, "Micro/nanofactory: concept and state of the art," in Proceedings of SPIE, Microrobotics and Microassembly II, vol. 4194, 2000.

[5] T. Gaugel, M. Bengel, and D. Malthan, "Building a mini-assembly system from technology construction kit," in Proceedings of the International Precision Assembly Seminar IPAS 2003, Bad Hofgastein, Austria, March 2003, AssemblyNET, 2003.

[6] S. Koelemeijer Chollet, L. Benmayor, J. Uehlinger, and J. Jacot, "Cost effective micro-system assembly automation," in Proceedings of the IEEE Emerging Technologies and Factory Automation, 1999.

[7] S. Koelemeijer Chollet, F. Bourgeois, C. Wulliens, and J. Jacot, "Cost modelling of microassembly," in Proceedings of the International Precision Assembly
Seminar IPAS 2003, Bad Hofgastein, Austria, March 2003, AssemblyNET, 2003.

[8] S. Koelemeijer Chollet, B. Moll, F. Bourgeois, C. Wulliens, L. Benmayor, and J. Jacot, "Small lot size micro-system assembly: the flexible microassembly cell," in Proceedings of the International Symposium on Robotics, Stockholm, Sweden, October 7-11, 2002, International Federation of Robotics, 2002.

[9] S. Koelemeijer Chollet, Méthodologie pour la conception de micro-systèmes et de leur équipement d'assemblage. Thèse no 2343, EPFL-IPR-LPM, Lausanne, 2001.

[10] G. Boothroyd and P. Dewhurst, Product design for assembly. Boothroyd Dewhurst Inc.: Wakefield, 1991.

[11] J. Bauer, "Ingénierie simultanée dans l'assemblage à hautes cadences de mini hat-parleurs," in Bulletin Infomat, 3 mai 2002, Neuchâtel, vol. 16, 2002.

[12] D. Becker and P. Sandborn, "On the use of yielded cost in modeling electronic assembly processes," IEEE Transactions on Electronics Packaging Manufacturing, vol. 24, pp. 195-202, July 2001.

[13] R. Wolfenbuttel, Silicon sensors and circuits, Onchip compatibility. London: Chapman Hall, 1996. 